\title{
Analytic Hitmap Equation of the Ideal Spherical Evaporator
}

\author{
B. P. Geiser, E. Oltman, D. J. Larson, T. R. Prosa, T. F. Kelly \\ Cameca Instruments Inc., 5500 Nobel Drive, Madison, WI 53711
}

Reconstruction of atom probe data is complicated by the role of the specimen (as the primary optic) in determining the trajectories of the evaporated atoms. If the specimen is non-isotropic with spatially coherent regions of differing evaporation fields, substantial non-uniformities may develop on the evaporating apex causing errors in the reconstructions [1]. One potential path toward improving reconstructions involves querying the spatial distribution of ion impacts on the detector and relating hitmap features to features present in the specimen [1,2]. Initial efforts in this area have already been published [3].

To develop a baseline understanding of the expected hitmap profile from evaporation that occurs without artifacts we examine the distribution of detector hits caused by the propagation of ions under ideal angular magnification when evaporated from a perfectly spherical apex. By considering how surface area elements on the spherical specimen apex (Figure 1) project onto the flat detector surface we observe that the radial hit density at a position $r_{D}$ on the detector a distance $\mathrm{L}$ from the specimen can be written as:

$$
d N\left(r_{D}\right)=\left(\frac{2 \pi \rho \xi R_{T}^{2}}{L}\right)\left\{1+K_{\alpha, f}\left(1-\frac{1}{\cos \left(\xi \theta_{D}\right)}\right)\right\} \sin \left(\xi \theta_{D}\right) \cos \left(\xi \theta_{D}\right) \cos ^{2}\left(\theta_{D}\right) d r_{D} d Z_{T}
$$

where

$$
\tan \left(\theta_{D}\right)=\frac{r_{D}}{L} \quad \text { and } \quad \theta_{T}=\xi \theta_{D}
$$

We use the $K_{\alpha, f}$ function for evolution of specimen radius with depth as described in [4] (generalized from $\mathrm{K}_{\alpha}$ as defined in [5]) and note that this expression applies to both the tangential and non-tangential apex geometries with zero or non-zero shank angles.

Approximation was required for the shank correction, so we compare the result to a Monte Carlo simulation where we incorporate the ideal projection law. We then repeatedly strip thin shells from the generated tip, using the $\mathrm{K}_{\alpha, \mathrm{f}}$ relationship for the shell shapes to maintain the desired tipshape. After projection, detected hits are accumulated in a hitmap (Figures 2a, 2c) and hits per unit radius are counted (Figures $2 \mathrm{~b}, 2 \mathrm{~d}$ ). The excellent agreement in both cases validates the approximations made in the derivation of our expression. Both the calculation and the simulation assume the correctness of the spherical apex model.

This baseline "projection hitmap" can be divided out of an experimental hitmap prior to extraction of local shape information based on hitmap densities resulting in a reduction of the mixing of projection effects into extracted tip-shapes.

\section{References}

[1] M. Miller and M.G. Hetherington, Surface Science, 246 (1-3) (1991) 442.

[2] E. A. Marquis et al., J. Microscopy (2011) doi: 10.1111/j.1365-2818.2010.03421.x.

[3] B. Gault et al., Ultramicroscopy 110 (9) 2010, 1215.

[4] D. Larson et al., "Non-Tangential Continuity Reconstruction in Atom Probe Tomography

Data" These Proceedings, (2011).

[5] D. Blavette et al., Revue Phys. Appl. 17 (1982) 435. 


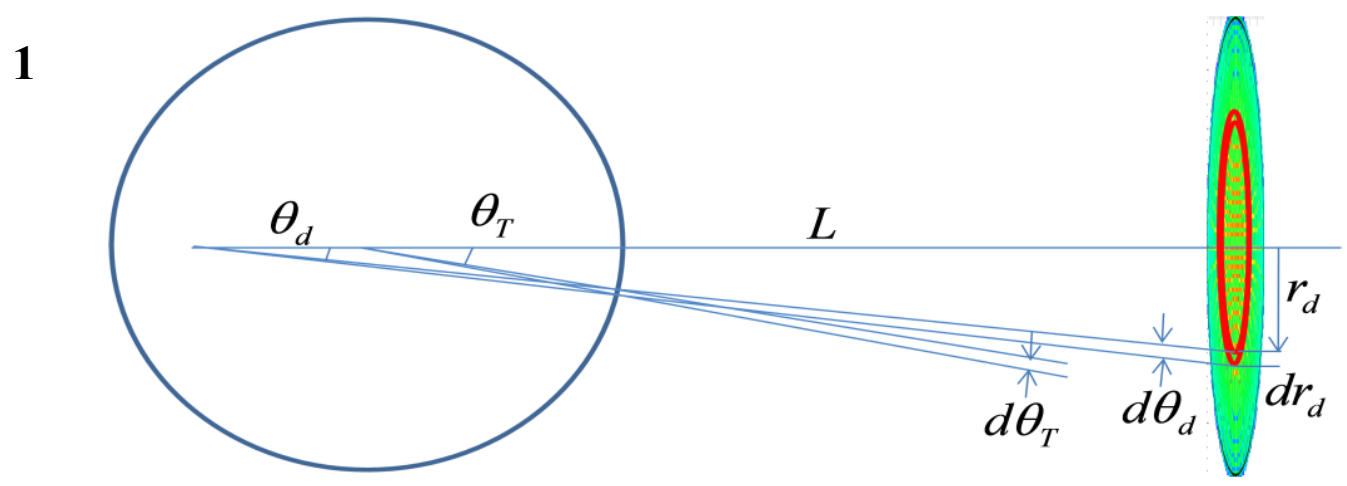

FIG. 1. Geometry describing the mapping of surface area elements on the spherical specimen apex onto a flat detector surface.

$2 \mathbf{a}$

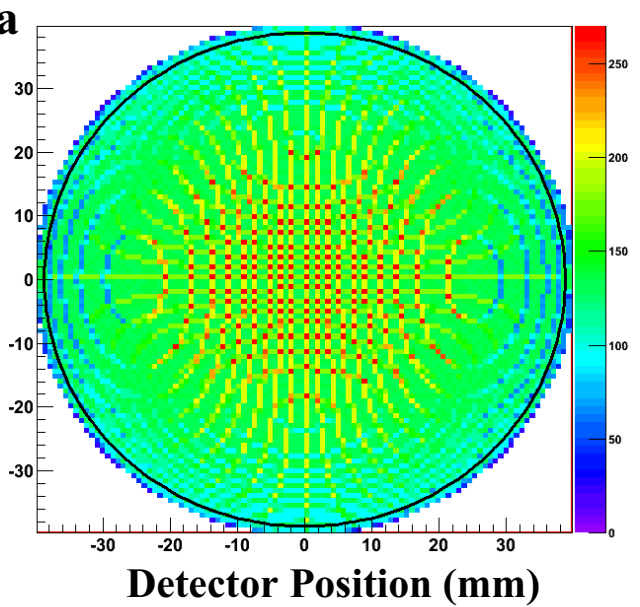

2c

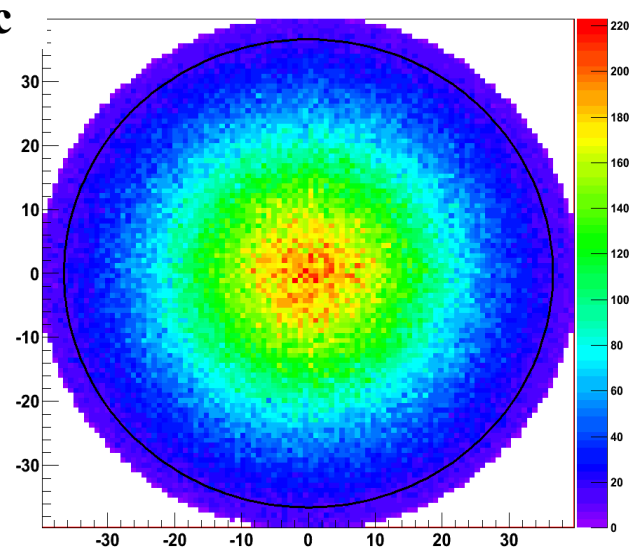

Detector Position (mm)

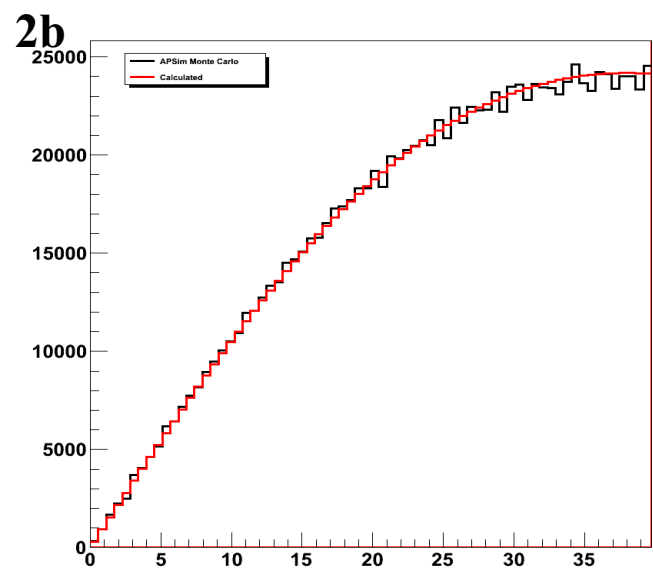

Detector Radial Position (mm)

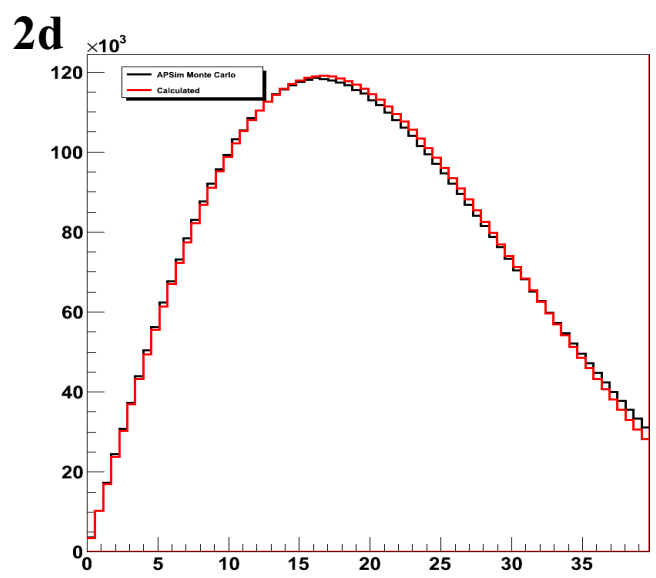

Detector Radial Position (mm)

FIG. 2.a) Generated hitmap for $80 \mathrm{~mm}$ flight-path, a shank angle of 0 degrees, angular magnification of 1.4 and $100 \%$ efficiency, b) Resulting radial distribution, c) Generated hitmap for $40 \mathrm{~mm}$ flight-path, a shank angle of 20 degrees, angular magnification of 1.4 and $50 \%$ efficiency, d) Resulting radial distribution. 